\title{
The feasibility of direct treatment planning via contrast-enhanced computed tomography: an evaluation of dose differences based on the dimensional dose distribution comparison method
}

\author{
Y. Li, X. Sun*, Q. Wang, Q. Zhou, B. Gu, H. Liu, G. Shi, D. Jiang, \\ T. Niu
}

Sir Run Shaw Hospital, Medical School of Zhejiang University, Hangzhou 310016, China

\begin{abstract}
- Original article

*Corresponding author:

Dr. Xiaonan Sun,

Fax: +8657186044817

E-mail: white_deer@263.net

Revised: May 2016

Accepted: July 2016

Int. J. Radiat. Res., April 2017; 15(2): $167-175$

DOI: 10.18869 /acadpub.ijrr.15.2.167

Background: We used a MapCHECK software-based dimensional dose distribution comparison method capable of evaluating point-to-point geometrical dose differences in volume to determine whether doses obtained from an enhanced computed tomography (CT)-based treatment plan, which better defines the target regions and organs at risk, differs from doses obtained from plain CT and then evaluated the feasibility of treatment planning via enhanced CT. Materials and Methods: Forty-three randomly selected patients underwent plain and subsequent enhanced CT with the same settings. Treatment plans developed for the two scans were identical in terms of planning parameters (e.g., isocentre, gantry angle, segments) and monitor units (MU) used for dose calculation. Horizontal and vertical dose distribution planes across the same isocentre were selected from two types of plan; a two-dimensional dose distribution analysis was used to determine the Distance-To-Agree (DTA) pass ratios of corresponding dose distribution planes. Results: Obtained doses at the head and neck (H\&N) and pelvic sites did not differ greatly between enhanced and plain CT. However, enhanced CT significantly influenced doses to the lower thoracic oesophagus. A corrected pass ratio that was achieved by non-pass points in lower isodose areas excluded from the statistical analysis had better clinical outcome. Conclusion: Radiation plans with multi-fields and multi-angles can reduce the influence of enhanced CT on torso cases and may even negate its influence on H\&N cases. Enhanced CT can be directly used for planning unless the target region contains the lower oesophagus and its surrounding blood vessel whose high density requires correction.
\end{abstract}

Keywords: Treatment planning, enhanced CT, plain CT, dimensional dose distribution, dose comparison.

\section{INTRODUCTION}

External beam radiotherapy dose calculation relies on electron densities that correspond to plain computed tomography (CT) values. Although enhanced CT provides clearer and more accurate definition and delineation of target and organ at risk (OAR), calculated doses based on these data that has higher electron densities in non-uniformly enhanced tissues and vessels lead to the potential delivery of excessive doses. Therefore, some institutes prefer to avoid enhanced CT during dose calculation (e.g., the Spain mode) (1). However, other researchers suggest that this effect is not obvious because the differences can be ignored or are clinically acceptable (2-6). Still other reports noted that large abdominal dose differences are influenced 
by the spleen; (2) accordingly, enhanced CT has been widely accepted for head and neck $(H \& N)$ planning.

Previously, phantoms or mathematical methods were often used in dose calculation studies (7-9). Contrast agent concentration calculations or high-density phantoms (used to mimic enhancement) help to clarify influences of these factors on dose calculation. Yamada and colleagues ${ }^{(7)}$ reported simulations of plain and enhanced CT by using water and iodine phantoms respectively. Meanwhile, he also reported a novel evaluation method, in which both of scanned images were imported to the Pinnacle planning system (Philips, Inc., Madison, WI, USA) and dose distributions were obtained from the identical single-beam radiation with subsequent MapCHECK (Sun Nuclear, Melbourne, FL, USA) dose difference verification. Ramm et al. (8) applied different density phantoms gotten by contrast agent flows with different velocities, which irradiated by various beam with different energies, they set up the quantification of potential variances in calculated doses. Robar et al. (9) examined the magnitude of tumour dose enhancement by the incidence on phantom containing different density contrast agent with simulation of various energy photon. Both groups suggested that influence of contrast agent on dose correlated with the beam energy, agent density, and concentration. Phantom-based methods simulate the ideal situations and verify the conceptualization and abstraction of actual situations. Unlike phantom material densities (usually distributed isotropically and uniformly), injected agents are distributed anisotropically in human bodies. Consequently, actual patients differ considerably from phantoms. Single-beam radiation differs considerably from the beams used in clinical settings.

Previous reports (2-6) described the use of tumours in specific sites to illustrate the influence of contrast agent. Shibamoto (2) conducted dose evaluations in the conventional anterior-posterior (AP) direction, but they were unable to reflect the multi-field/multi-angle feature used in intensity-modulated radiotherapy (IMRT). The method used in those studies did not reflect the multi-beam and multi-angle set-up used in IMRT. IMRT plan involves many organs, many different fields and many differential density distribution issues. IMRT planning has become standard for treatment planning, its optimization, which results in different beam directions and weights, can dilute the effect from above-described ideal simple-field methods with uniform high phantom densities. Choi et al., (3) Lee etal.,(4) Liauw etal., (5) and Xiao etal. (6) used IMRT to evaluate lung cancer, H\&N cancers, and nasopharyngeal carcinoma (NPC). However, statistical comparisons of inter-plan dose variances in the above-mentioned studies (2-6) relied on dose volume histograms (DVHs) and statistical dose distribution parameters. For example, DVH statistical parameters such as the maximum dose $\left(D_{\max }\right)$, average dose $\left(D_{\text {mean }}\right)$, volume doses $\left(D_{95}, D_{50}\right)$, and dose volumes $\left(V_{20}\right.$, $\mathrm{V}_{30}$ ) cannot be used to assess the geometric accuracy of a non-uniform dose distribution because spatial information is lost. Thus, the DVH does not reflect the two or three-dimensional dose distribution. Accordingly, we describe a method for planning and evaluating non-uniform treatment doses that consider the geometric position.

The distance-to-agreement (DTA) pass ratio can be used to apply for dose distribution comparisons by its straightforward point-topoint dose verification on geometric position. Yamada and colleagues (7) applied a two-dimensional dose distribution analysis tool in MapCHECK software with a DTA comprising a dose threshold of $10 \%$, an acceptance criteria percent difference of $2 \%$,and distance of $2 \mathrm{~mm}$ $(10 \%, 2,2$; threshold is the per cent contour above which all points are included in the DTA analysis; per cent difference is the allowed difference between co-located compared points; distance is the radius around the compared point definitions of values)to compare the dose distribution by outcome of pass ratio. However, results of mono-beam and phantom-based evaluations vary from those in actual clinical settings, as patient treatment requires greater complexity. Mono-beam radiation tests with phantoms which simulate uniform contrast 
agent concentrations yields dramatic dose differences, it is not consistent with those observed in real patients, in whom tissue density distributions differ and multi-field and multi-angle radiation fields are used for planning.

Shibamoto and colleagues ${ }^{2}$ also mentioned that during patient treatment planning, the influence of contrast agent on dose was tumour site-dependent. However, the planning process in that study mainly relied on three-dimensional conformal radiotherapy (3DCRT), for which plans are constructed using a few radiation fields, only a few reference points are used to compare differences in monitor units (MUs). IMRT planning design is not considered. As IMRT is the most popular radiotherapy technique, $88.37 \%$ of samples used to determine dose distribution in our study were based on IMRT plans. For dose variance evaluation, we used the MapCHECK two-dimensional dose distribution analysis software package to compare dose distribution differences between the two orthogonal dose planes.

\section{MATERIALS AND METHODS}

In our method, the patient underwent an initial plain CT scan (SOMATOM Definition AS; SIEMENS, Munich, Germany). Subsequently, an iodine-based enhancing agent (Iopamiro 370, 90 $\mathrm{ml}$; Bracco, Shanghai, China) was injected intravenously prior to enhance CT, during which the same body site was imaged in the venous phase. For patients undergoing H\&N scans, a thermal mask was used to acquire 3-mm-thick image slices. For other sites (e.g.: thorax, abdomen, and pelvis), a skin surface marker was used and 5-mm-thick image slices were acquired (exception: 2-mm-thick slices for the prostate). The delay times for the $H \& N$, thoracic, abdominal, and pelvic regions were $65 \mathrm{~s}, 38 \mathrm{~s}$, $60 \mathrm{~s}$, and $65 \mathrm{~s}$, respectively. The present study was approved by the ethics committee of the Sir Run Shaw Hospital (Hangzhou, China). Plain and enhanced CT images were imported in Digital Imaging and Communications in Medicine (DICOM) format from the network into a
Pinnacle 7.6 treatment planning system (Philips, Inc.), thus allowing target region and organs at risk (OAR) definition and contouring by physician. Specifically, direct machine parameter optimization (DMPO) and a convolution/ superposition algorithm were applied, and a minimum constrained radiation field of $10 \mathrm{~cm}^{2}$ was defined during IMRT optimization. Beam energy with $6 \mathrm{MV}$ is used for $\mathrm{H} \& \mathrm{~N}$ calculations and $10 \mathrm{MV}$ for others. Treatment planning continued until the physician was satisfied that the dose distribution met the clinical treatment requirement. The same plan parameters (coordinate system, beam angle, multileaf collimator [MLC] segment shape, dose calculation range, calculation grid size, MU values, and dose prescription) and same isocentre point (ISO) were used for plain and enhanced CT. Dose calculations based on the above-mentioned plan settings were performed by using another CT image. The applied average radiation field number, average segment number, and radiation techniques (e.g., IMRT or 3DCRT) used for planning were classified according to the $\mathrm{H} \& \mathrm{~N}$, thoracic/abdominal, and pelvic regions and are summarized in table 1 .

Although scanning was performed while the patient was in a resting position, the positions used for plain and enhanced CT differed slightly. During thoracic and upper abdominal scanning, respiratory motion lifts the target region and displaces the OAR. Similarly, cardiac motion and gastrointestinal motility affect the position of the adjacent target region. In contrast, the effects of respiration and organ movement on the $\mathrm{H} \& \mathrm{~N}$ and pelvic regions are relatively small. The main blood vessels are located in the thoracic and abdominal region, causing increased contrast agent uptake. In contrast, the smaller vessels in the H\&N and pelvic regions exhibit less contrast agent uptake. To take into account this discrepancy, we classified patients into $\mathrm{H} \& \mathrm{~N}$, thoracic/abdominal, and pelvic groups for our analysis.

The plain and enhanced CT results could be compared objectively, as all treatment planning parameters were identical. Hence, the potential agent density-based dose difference between plain and enhanced CT could be compared. We 
first calculated the two-dimensional orthogonal (they have smallest mutual correlation, and are chosen to represent the three-dimensional distribution), horizontal(x-plane), and vertical (y-plane) plane dose distributions across the same ISO and entered these values into MapCHECK to evaluate differences in plan dose distributions. The DTA pass ratio, with the above -described settings of $10 \%, 2$, 2, was applied to compare dose distributions. Data in table 1 was analysed by comparing means function in Statistical software SPSS13.0 (SPSS Inc., Chicago, IL, USA).

Table 1. Statistical data of samples from the head and neck, thoracic/abdominal, and pelvic regions.

\begin{tabular}{|c|c|c|c|c|c|c|}
\hline & \multirow[t]{2}{*}{$\begin{array}{c}\text { Number } \\
\text { of beams } \ddagger\end{array}$} & \multirow[t]{2}{*}{$\begin{array}{l}\text { Numbers of } \\
\text { segments }\end{array}$} & \multirow{2}{*}{$\begin{array}{c}\text { Horizontal dose planar } \\
\text { comparison pass ratio } \\
\text { (corrected) }\end{array}$} & \multirow[t]{2}{*}{$\begin{array}{c}\text { Vertical dose planar } \\
\text { comparison pass ratio } \\
\text { (corrected) }\end{array}$} & \multicolumn{2}{|c|}{$\begin{array}{c}\text { Differences in SSDst between } \\
\text { corresponding beams from the } \\
\text { two plans }(\mathrm{cm})\end{array}$} \\
\hline & & & & & Each beam & Each patient \\
\hline $\begin{array}{l}\text { Head and neck } \\
(n=6,1,5) \S\end{array}$ & $5.83 \pm 2.71$ & $23.83 \pm 17.02$ & $\begin{array}{c}99.52 \pm 0.69 \\
(99.72 \pm 0.52)\end{array}$ & $\begin{array}{c}99.58 \pm 0.61 \\
(99.85 \pm 0.25)\end{array}$ & $0.01 \pm 0.24$ & $0.01 \pm 0.09$ \\
\hline $\begin{array}{l}\text { Thoracic and abdominal } \\
\qquad(n=25,1,24)\end{array}$ & $5.24 \pm 0.78$ & $21.24 \pm 7.85$ & $\begin{array}{c}98.06 \pm 2.27 \\
(99.18 \pm 1.15)\end{array}$ & $\begin{array}{c}97.94 \pm 2.54 \\
(99.55 \pm 0.91)\end{array}$ & $0.45 \pm 2.18$ & $0.49 \pm 1.58$ \\
\hline $\begin{array}{c}\text { Pelvic } \\
(n=12,3,9)\end{array}$ & $5.00 \pm 1.04$ & $14.75 \pm 8.35$ & $\begin{array}{c}99.33 \pm 1.26 \\
(99.96 \pm 0.14)\end{array}$ & $\begin{array}{c}98.59 \pm 2.46 \\
(99.85 \pm 0.49)\end{array}$ & $0.16 \pm 0.88$ & $0.17 \pm 0.35$ \\
\hline \multirow{2}{*}{ Total } & \multirow{2}{*}{$5.26 \pm 1.26$} & \multirow{2}{*}{$19.79 \pm 9.94$} & $\begin{array}{c}98.62 \pm 1.96 \\
(99.47 \pm 0.96)\end{array}$ & $\begin{array}{c}98.35 \pm 2.38 \\
(99.68 \pm 0.75) \\
\end{array}$ & & \\
\hline & & & \multicolumn{2}{|c|}{$\begin{array}{c}98.48 \pm 2.17 \\
(99.57 \pm 0.86)\end{array}$} & & \\
\hline
\end{tabular}

+ SSD, source-skin distance

¥ Data are shown as means \pm standard deviations.

$\S$ For $n=(a, a 1, a 2)$, a, indicates the number of samples, a1 indicates the number treated using 3-dimensional conformal radiotherapy, and 22 indicates the number treated using intensity-modulated radiotherapy.

\section{RESULTS}

Figure 1A shows the vertical dose cross-section distribution of a thoracic oesophageal cancer patient; in this figure, green points indicate the reference points, and red and blue points respectively indicate higher and lower dose points relative to the reference point. Accordingly, this cross-section indicates a distinct difference in dose distribution, with a pass ratio of $91.4 \%$. However, these differences existed mainly in the surrounding low-dose region, which has only a minor influence on clinical treatment outcome. In other words, these differences are also largely site-dependent, which might explain the relatively low pass ratio for the thoracic/ abdominal region. So we have a corrected pass ratio concept.it can be obtained by doing so: if the position of the non-pass dose point, lies outside of the $70 \%$ ISO-dose line, they would be rejected and not calculated in pass ratio. Only different points in the target region and high dose region $(\geq 70 \%)$ are considered for calculating. Then the pass ratio in figure 1-A might reach as high as $99.9 \%$, which we think as a corrected pass ratio. Figure 1-B presents a dose view of a lower abdomen with a high pass ratio of $99.4 \%$, similar to the case in Figure 1- A, 100\% pass ratio was achieved after correction.

For each patient and radiation field, source-skin distances (SSDs) from the corresponding radiation fields were recorded for both CT techniques and subjected to a simple statistical analysis in order to evaluate body position displacement in the $\mathrm{H} \& \mathrm{~N}$, thoracic/ abdominal, and pelvic regions. The pass ratios (and corrected pass ratios) of the two-dimensional dose distributions in the two orthogonal planes were also analysed (table 1). 


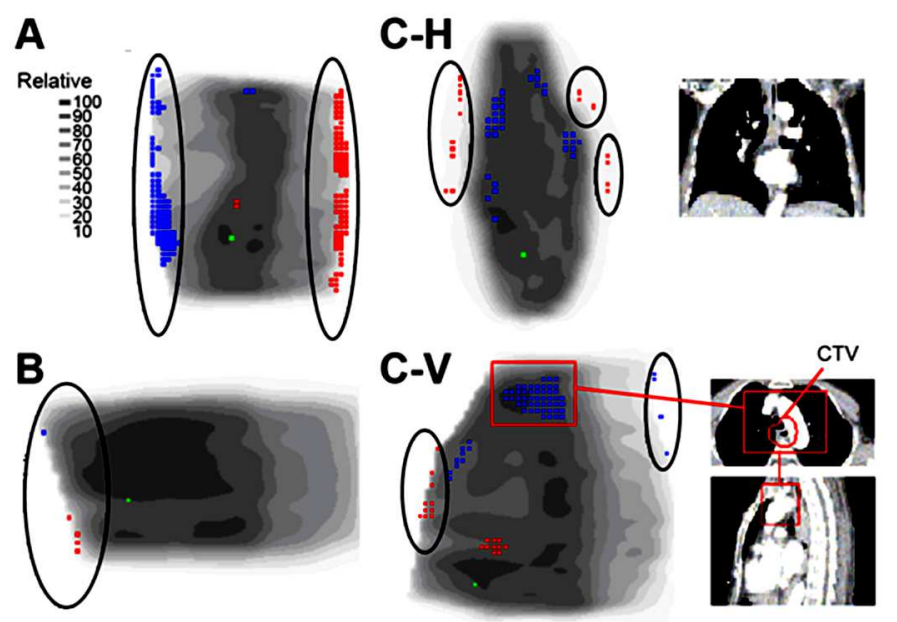

Figure 1. (A) Vertical dose distribution section view of a thoracic oesophageal case. (B)Vertical dose distribution section view of Lower abdominal case(C-H)/(C-V) Horizontal and vertical dose distribution sections view of the lower oesophageal case. The scale indicates relative contrast. Points in black ellipsoids, which are non-pass and outdise of the $70 \%$ isoline areas are not included in corrective pass ratio calculations. Points in red boxes, which are non-pass and inside of the $70 \%$ isoline areas, locate in the areas with high density. CTV, clinical target volume.

\section{DISCUSSION}

Yamada and colleagues ${ }^{(7)}$ reported that the mono-beam radiation pass ratio for enhanced CT was $50-60 \%$ less than that for plain CT. In contrast, our results indicated a similar pass rate between enhanced and plain CT $(98.48 \pm 2.17 \%)$ for actual clinical cases (table 1). Our convolution/superposition algorithm (TPS dose calculation methods) is superior in terms of the calculation error, (10-12) although a 2-3.6\% calculation error remained. With regard to TPS calculation error, this rate was clinically acceptable. Through pass ratio analysis and its correction we determined that enhanced CT might be clinically acceptable for treatment planning (table 1).

Figure 1-C-H and 1-C-V illustrate the horizontal and vertical sections from a patient with lower oesophageal cancer; in this typical case, the dose differences were more distinct in both sections. The pass ratios of the horizontal and vertical sections were $94.4 \%$ and $95.8 \%$ respectively. The radiation target region contained not only the mid-lower oesophageal segment, but also part of the vascular system, in which the tumour had grown. Accordingly, the clinical target volume (CTV) included vascular tissue, as indicated by the National Comprehensive Cancer Network (NCCN) 171 guideline for oesophageal cancer. The CTV was surrounded by the heart and primary vessels, which had a high contrast agent density, resulting in a low pass ratio in this region. Figure 2 illustrates CT value distributions that crossed the ISO-centre in the anterior-posterior (AP) and left-right (LR) directions. The heart in figure 2 was indicated by a region with a high-density difference. The upper left panel of figure 2 shows the radiation field settings with beam passes from the high-density region. Beam angles were restricted by the dose constraint set for the lung. All beams, especially higher weight beams (e.g., beam 2, 33.86\% and beam 4, $25.48 \%$ ) passed through high agent density regions within the radiation fields. The dose in the high agent density region induced by enhanced CT differed considerably from that induced by plain CT. Accordingly, non-pass points were concentrated in the target region and the surrounding high agent density areas. As these affected regions were located inside of the $70 \%$ ISO-dose lines areas, the corrected horizontal and vertical sections pass ratios (96.24\% and $96.25 \%$, respectively) were not distinct to become better. Therefore, particular attention should be paid to treatment planning for target regions with density changes based on enhanced CT.

Int. J. Radiat. Res., Vol. 15 No. 2, April 2017 

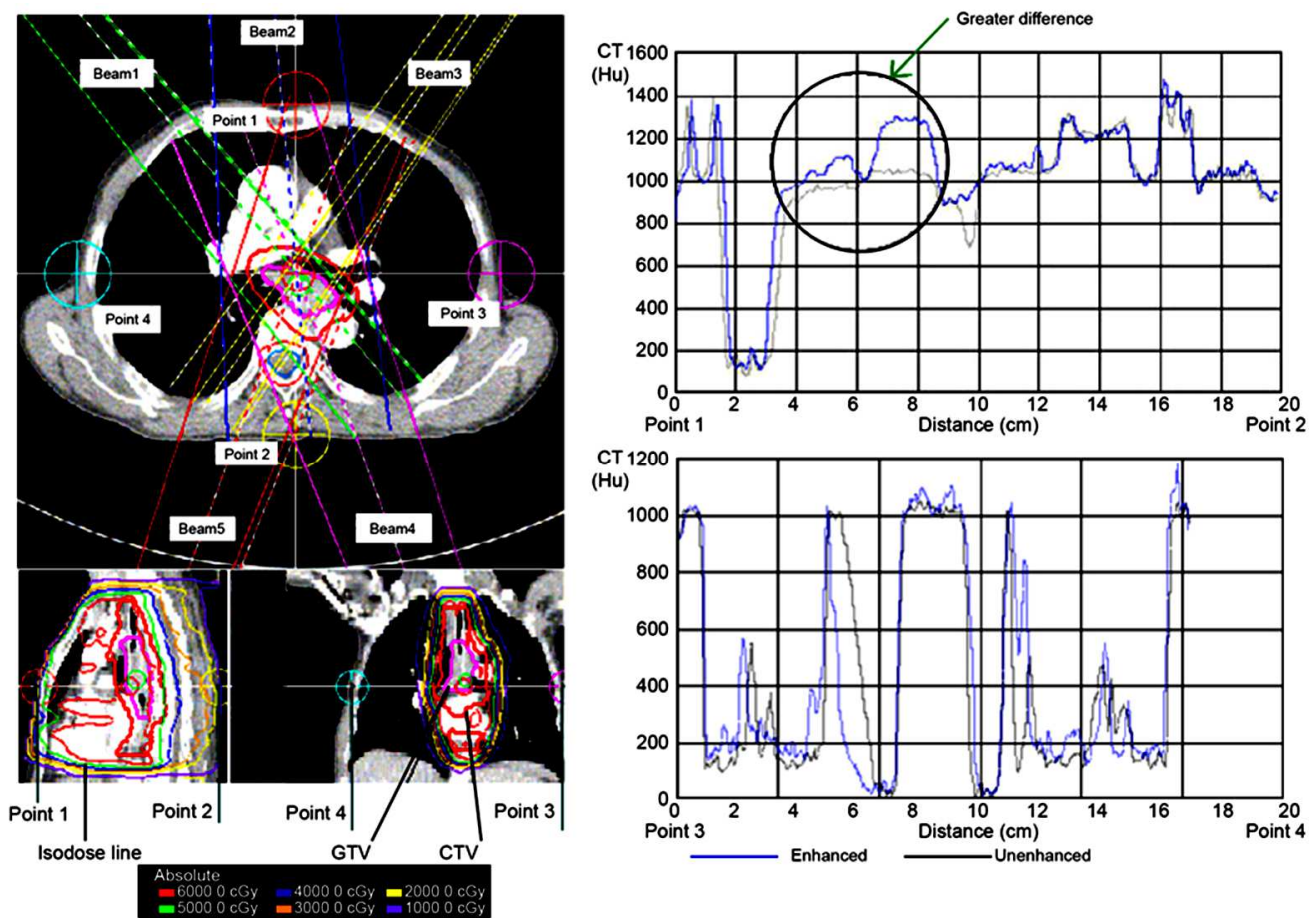

Figure 2. Computed tomography (CT) value distributions in the superior-posterior and left-right directions across the isocentre. CTV, clinical target volume; GTV, gross target volume.

When data from all patients were corrected, nearly all plans achieved high pass ratios, with an average of $99.57 \% \pm 0.86 \%$ (table 1 ). This improvement was particularly obvious in the pelvic region, possibly because the non-pass points in this region were mainly located at the body surface rather than in the target region. This significant improvement in the pass ratio made our conclusion more easily acceptable.

The value of the pass ratio also correlated with patient motion. For example, respiratory motion and consequent surface displacement caused lower pass ratios in the thoracic/ abdominal region, comparing to the pelvic region and the $H \& N$ region, which had the smallest displacement(nearly rigid)(table 1). However, pass ratio correction mitigated this tendency, indicating 1) the robustness of target region ISO-centre beam irradiation regardless of position error and 2) a smaller dose influence from enhanced CT than the current data. The pass ratio will increase if the position errors decrease.

The $H \& N$ and pelvic regions contain smaller vessels and are thus less affected by contrast agents with corresponding smaller effects from density differences. In contrast, organs such as the kidneys, heart, liver, spleen, and primary vessels in the thoracic/abdominal region are strongly affected by agent density with corresponding dose heterogeneity. The results in Table 1 support this concept. The $\mathrm{H} \& \mathrm{~N}$ region features dual radiation fields (e.g., LR and right-left [RL] direction beams) and three-field IMRT. However, these factors do not greatly affect dose calculation errors. No obvious agent density-related dose differences were observed with IMRT in the thoracic/abdominal and pelvic regions; if there is any difference, the difference observed was only slightly larger than that of the H\&N.

For phantom-based enhanced CT studies, the planned radiation beam is limited to one or two angles and the field area is larger. These factors increase dose differences in an agent density-dependent manner. This dose difference is lower for IMRT plans involving multi-angle beams and multi-segment fields (some with small areas) to ensure a multi-field overlap irradiation. Our results were consistent with 
those reported by Yamada et al.(7) and Ramm et al. (8LYamada and colleagues suggested that small radiation fields were not strongly affected by the contrast agent and thus had high pass ratios (7). Based on a mono-field radiation phantom study, Ramm and colleagues reported that the dose difference between enhanced and plain CT at an energy beam of $6-25 \mathrm{MV}$ was proportional to the agent density and velocity (8). This dose disturbance was $<1-3 \%$ at $<500$ Hounsfield Units (HU) and a field radius of $<5 \mathrm{~cm}$. A small field with lower density contrast agent had only a small influence on the dose, and multi -field radiation could further reduce the effects of the contrast agent. Both of these studies observed that a high-MV beam was more resistant to contrast agent and was associated with smaller dose differences $(7,8)$. Liauwand colleagues concluded that a multi-field, multi-angle region with a small radiation volume would experience a smaller dose disturbance (5). These conclusions could explain our IMRT-based results. For enhanced CT, factors such as a high-density tissue distribution, relative position to the target region, radiation angle, and high-density volume through which the beam passes could all affect dose calculations. However, this influence is not dramatic if multi-field (including segments) and multi-angle irradiation techniques (e.g. IMRT) are used. Yamada and colleagues also reported the achievement of a high pass ratio when evaluating a small field using MapCHECK during a phantom study, (7) indicating that the influence of contrast agent on dose is not obvious in small radiation fields. Although Ramm and colleagues agreed with this concept (8), Xiao and colleagues disagreed (6). One possible reason for this discrepancy is the use of different design and evaluation methods. The DVH-based statistical dose evaluation method did not consider the large uncertainty of the exact geometrical position with respect to dose evaluation. Xiao and colleagues focused only on lung tissue; therefore, further studies are required for verification.

Comparing to previous studies, we did not use image fusion/registration $(3,4,6)$ as the patient position we considered reflected the actual treatment situation. The two CT scanning times (including the agent injection time) were shorter than the IMRT irradiation time. The displacement in patient position during the scanning period did not exceed the displacement during the treatment stage. It is more clinically relevant and practical to perform dose comparisons using actual patient positions. Deformable image registration (DIR) of organ and target regions is possible, but whether this method can accurately reflect the effect of the actual body position during treatment should be carefully considered, especially for the target region and OAR. Because the DIR always contains error (approximate average DIR error: $1.44 \mathrm{~mm})^{(13)}$, Kirby and colleagues suggested that different DIRs would correspond to different clinical applications and that trade-off points exist for the errors in volume and similarity matching (13). Notably, the CT position used for DIR and the final fused position also differ in terms of phase motion. It remains uncertain whether the fused position is the true radiation position or, at least, the best approximation. Currently, dose pass rate evaluations are conducted precisely and directly and involve fewer manipulations of the data and patient position to better approximate an actual treatment situation.

The dimensional dose distribution comparison method permits straight forward point-to-point comparisons in dose planes and is thus rational and objective. However, no extractable parameters are correlated with evaluations of the OAR response and treatment effect. Hence, further study is needed.

Further investigation is also needed to determine whether the influence of an enhanced CT-based dose calculation will be smaller in IMRT plans containing higher numbers of fields or angles. Virtual or artificial intelligence-based technology methods to reduce dose disturbances in tumour regions (e.g., the lower oesophagus) and thus remove the enhancement effect are also needed (7). Simple methods $(14,15)$ that incorporate density correction could be applied for clinical corrected dose calculations. for example, the HU values of the heart and great vessels in the enhanced CT were replaced by 
average $\mathrm{HU}$ values obtained from the unenhanced $\mathrm{CT}$, and in the Lung patients the CT to density conversion table used clinically was altered to reduce the effect of the increased density due to the contrast agent, in which tissues with a density between $1 \mathrm{~g} \cdot \mathrm{cm}^{-3}$ and 1.2 $\mathrm{g} \cdot \mathrm{cm}^{-3}$ were set equal to a density of $1 \mathrm{~g} \cdot \mathrm{cm}^{-3}$. In comparison to the work conducted by Shibamoto and colleagues, (2) who used AP, RL, RA-LP oblique, and RA-LP oblique directional radiation, we used IMRT with an increased number of radiation directions and large weighed radiation fields at other angles. Hence, our dose disturbances in the spleen and kidney were less obvious than those reported previously (2). Using our technique, the radiation field was more dispersed and the beam field did not deliberately pass through the enhanced OAR. The upper abdominal sample number in this study was small $(n=8)$, However, more additional data are needed to draw a solid conclusion.

Despite the existence of position errors, our evaluation more closely approximates actual clinical situations; our evaluation is useful with respect to the clinical endpoint. More accurate evaluation of dose differences is possible if respiration gating or other novel image-guided radiotherapy techniques are applied to control respiration-induced organ motion, thus reducing positioning errors (6).

Our results achieved in the H\&N region were consistent with those from earlier studies, (2-6) thus supporting the universal agreement that enhanced CT-induced dose disturbance scan be ignored in this region. Similarly, these dose disturbances do not have obvious effects on the pelvic region. Effects of dose disturbances on abdominal organs (e.g., liver, kidney, and spleen) and the thorax are small and clinically acceptable. However, a large dose disturbance is induced by contrast agents in the lower oesophageal segment of the inferior thoracic region, which is surrounded by high agent density organs such as the heart and primary vessels. The pass ratio can be improved by doing correction. Hence, in actual clinical cases, enhanced CT can be used directly for IMRT planning, except for patients in whom the target

Int. J. Radiat. Res., Vol. 15 No. 2, April 2017 region contains the lower oesophageal segment and surrounding blood vessels, as this high density area requires density correction.

\section{ACKNOWLEDGEMENTS}

This work was supported in part by a grant from the Major Science and Technology Program of Zhejiang Province in China (No.2013C03044-6).

Conflicts of interest: Declared none.

\section{REFERENCES}

1. Casas F, Vinolas N, Sanchez-Reyes A, Jorcano S,Planas I, Marruecos J, Pino F, Herreros A,Biete A (2006) Spanish patterns of care for $3 D$ radiotherapy in non-small-cell lung cancer. Int J Radiat Oncol Biol Phys, 65: 138-42.

2. Shibamoto $Y$, Naruse A, Fukuma $H$, Ayakawa $S$, Sugie $C$, Tomita N (2007) Influence of contrast materials on dose calculation in radiotherapy planning using computed tomography for tumors at various anatomical regions: a prospective study. Radiother Oncol, 84: 52-5.

3. Choi Y, Kim JK, Lee HS, Hur WJ, Hong YS, ParkS, AhnS, $\mathrm{ChoH}$ (2006) Influence of intravenous contrast agent on dose calculations of intensity modulated radiation therapy plans for head and neck cancer. Radiother Oncol, 81: 15862.

4. Lee FK, Chan CC, Law CK (2009) Influence of CT contrast agent on dose calculation of intensity modulated radiation therapy plan for nasopharyngeal carcinoma. J Med Imaging Radiat Oncol, 53: 114-8.

5. Liauw SL, Amdur RJ, Mendenhall WM, Palta J, Kim S (2005) The effect of intravenous contrast on intensity-modulated radiation therapy dose calculations for head and neck cancer. Am J Clin Oncol, 28: 456-9.

6. Xiao JH, Zhang H, Gong YL, Fu YC, Tang Bin, Wang SC, Jiang QF, Li P (2010) Feasibility of using intravenous contrastenhanced computed tomography (CT) scans in lung cancer treatment planning. Radiother Oncol, 96: 73-7.

7. Yamada S, Ueguchi T, Ogata TMizuno H, Ogihara R, Koizumi M, Shimazu T, Murase K, Ogawa K (2014) Radiotherapy treatment planning with contrast-enhanced computed tomography: feasibility of dual-energy virtual unenhanced imaging for improved dose calculations. Radiat Oncol, 9: 168.

8. Ramm U, Damrau M, Mose S, Manegold KH, Rahl CG, Böttcher HD (2001) Influence of CT contrast agents on dose calculations in a 3D treatment planning system. Phys Med Biol, 46: 2631-5.

9. RobarJL, Ricco SA, Martin MA (2002) Tumor dose enhancement using modified megavoltage photon beams and 
contrast media. Phys Med Biol, 47: 2433-49.

10. Fogliata A, Vanetti E, Albers D, Brink C, Clivio A, Knöös T, Nicolini G, Cozzi L (2007) On the dosimetric behaviour of photon dose calculation algorithms in the presence of simple geometric heterogeneities: comparison with Monte Carlo calculations. Phys Med Biol, 52: 1363-85.

11. Vanderstraeten B, Reynaert N, Paelinck L, Madanil, Wagter CD, Gersem WD, Neve WD, Thierens H (2006) Accuracy of patient dose calculation for lung IMRT: A comparison of Monte Carlo, convolution/superposition, and pencil beam computations. Med Phys, 33: 3149-58.

12. Paelinck L, Reynaert N, Thierens H, Neve WD, Wagter CD (2005) Experimental verification of lung dose with radiochromic film: comparison with Monte Carlo simulations and commercially available treatment planning systems. Phys Med Biol, 50: 2055-69.

13. Kirby N, Chuang C, Ueda U, Pouliot J (2013) The need for application-based adaptation of deformable image registration. Med Phys, 40: 011702.

14. Hwang UJ, Shin DH, Kim TH, Moon SH, Lim YK, Jeong H, Rah JE, Kim SS, Kim JY, Kim DY, Park SY, Cho KH (2011) The effect of a contrast agent on proton beam range in radiotherapy planning using computed tomography for patients with locoregionally advanced lung cancer. Int J Radiat Oncol Biol Phys, 81: 317-24.

15. Burridge NA, Rowbottom CG, Burt PA (2006) Effect of contrast enhanced CT scans on heterogeneity corrected dose computations in the lung. J Appl Clin Med Phys, 7: 1-12 
